Article

\title{
Nutritional Stress Causes Heterogeneous Relationships with Multi-Trait FA in Lesser Black-Backed Gull Chicks: An Aviary Experiment
}

\author{
Trisha Gupta ${ }^{1, *,+}{ }^{\dagger}$ Cátia S. A. Santos ${ }^{1,2,+}$, Alejandro Sotillo ${ }^{1,2}$, Liesbeth De Neve ${ }^{1}$, \\ Eric W. M. Stienen ${ }^{3}$, Wendt Müller ${ }^{4}$ and Luc Lens ${ }^{1}$ \\ 1 Terrestrial Ecology Unit (TEREC), Ghent University, 9000 Ghent, Belgium; catiasantos@ua.pt (C.S.A.S.); \\ alejandro.sotillogonzales@ugent.be (A.S.); liesbeth.deneve@ugent.be (L.D.N.); luc.lens@ugent.be (L.L.) \\ 2 Department of Biology \& CESAM, University of Aveiro, Campus de Santiago, 3810-193 Aveiro, Portugal \\ 3 Research Institute for Nature and Forest (INBO), 1070 Brussels, Belgium; eric.stienen@inbo.be \\ 4 Ethology (ETHO), University of Antwerp, 2000 Antwerp, Belgium; wendt.mueller@uantwerpen.be \\ * Correspondence: trishagupta0405@gmail.com; Tel.: +32-9-2645254 \\ $\dagger$ These authors contributed equally to this work.
}

Academic Editor: John H. Graham

Received: 31 August 2016; Accepted: 11 November 2016; Published: 17 November 2016

\begin{abstract}
Environmental stressors have the potential to induce perturbations in the development of young individuals, leading to aberrant and unstable development. This may manifest as fluctuating asymmetry (FA; small, non-directional changes in the bilateral symmetry of morphological traits). Although widely regarded as a proxy for stress effects, the use of FA as a biomarker is still a topic of much debate. We investigated the applicability of FA as an indicator of nutritional stress (brought about by energetic constraints) by experimental manipulation of the diet composition and quantity during the growth of Lesser Black-backed Gull (Larus fuscus) chicks. FA as an endpoint was measured across the tarsus, wing and 10th primary feather when chicks reached 30 days of age. Although levels of asymmetry were found to increase with stress in the feather, relationships with tarsus and wing FA were mixed and mostly non-significant. Furthermore, we did not find any correlations in unsigned FA between traits, indicating the absence of organism-wide asymmetry. Our study was therefore unable to find unequivocal evidence in support of the application of FA as a reliable estimator of nutritional stress.
\end{abstract}

Keywords: fluctuating asymmetry; Larus fuscus; nutritional stress; energetic constraints; biomarkers

\section{Introduction}

Natural populations of plants and animals face increasing levels of environmental stress due to anthropogenic activities, and there is hence a growing need to monitor these stress impacts. Biomarkers, defined as functional measures of exposure to stressors [1], are important tools in this respect, developed and applied by ecologists for the conservation and management of biological systems. Fluctuating asymmetry (FA), which refers to small, random deviations from perfect symmetry in bilateral traits [2], is one commonly used marker of stress. Since both sides of a bilateral trait are normally under the control of the same genome and exposed to the same environment, FA results from the inability of an individual to buffer against perturbations during its development [3]. These perturbations act locally at a cellular level, their effects accumulating on the left and right sides separately to bring about aberrant growth and asymmetry [4]. A high level of FA may therefore indicate exposure to stress, which led to the loss of developmental stability [5].

FA as a marker of stress has been credited with being low cost, non-lethal and easy to use [6,7]; moreover, it is one of the few markers where the norm (i.e., perfect symmetry) is known [8]. It is also 
widely assumed to be negatively related to components of fitness such as growth and longevity [6]. Furthermore, FA has been proposed to have a higher sensitivity to stress effects than these fitness components, making it detectable in individuals in response to stress before the corresponding decrease in fitness [9]. It therefore has the potential to be an early warning system for the implementation of mitigation measures [10]. However, the application of FA as a biomarker is still under much debate and controversy. While several hypotheses have been proposed, the underlying mechanisms producing developmental instability and FA are yet to be completely understood [6]. This might be one reason why relationships between FA, stress and fitness components have often been found to be trait, stress or organism-specific [11], with varied and often contrasting results obtained across different studies (see [12]). FA measured on a single trait is also shown to have weak and heterogeneous relationships with stress [13].

Despite these drawbacks, FA continues to be a popular indicator of stress and proxy for fitness, and has been used across various animal groups. For birds, nutritional stress during development can incur an important fitness cost; it may create energetic deficits in an individual, leading to a scenario where growth and other more vital functions are prioritized over developmental stability [14]. Food deprivations have hence been associated with higher levels of asymmetry in various bird species [14-16]. Poor diet quality with nutritive deficiencies may also lead to increased FA [17]. As stated before, however, FA-stress relationships are not always consistent; Vangestel and Lens [18], for instance, did not find increased FA levels in the tarsus and rectrix of House Sparrows (Passer domesticus) under nutritional stress. Clearly, more research is needed; as correlational field studies can be confounded by uncontrolled environmental factors, experimental manipulations of stress may be a more powerful method for the study of FA.

This paper experimentally assesses the suitability of FA as a biomarker of nutritional stress, particularly in young seabirds. Food stress has become especially relevant for this group of birds in European seas in light of the recent reform of the EU Common Fisheries Policy [19], aimed at gradually phasing out the practice of discarding bycatch by fishing vessels. This 'discards ban' could affect populations of scavenging seabirds that are dependent on fishery discards as a food source [20]. Preliminary studies have suggested that lowered availability of discards is likely to produce an overall food shortage, which may result in an energetic stress in the absence of sufficient alternatives [21]. In this context, the scavenging Lesser Black-backed Gull (Larus fuscus) is an ideal model for the study of nutritional stress effects. This species is a generalist feeder, foraging on offshore surface prey like fish and crustaceans as well as terrestrial food and urban refuse [22]. Individuals, however, have shown varying degrees of specialisation in their feeding habits [23], which means that these dietary shifts and restrictions are likely to have a spectrum of effects across individuals.

An aviary experiment was conducted during the 2015 breeding season where newly hatched L. fuscus chicks were reared on a range of diets expected to induce different degrees of nutritional stress through their different energetic values. Fluctuating asymmetry was then assessed for each chick at the end of the experiment by measuring bilateral differences in three morphological traits: the tarsus, wing and primary feather, analysed both independently and combined as multi-trait FA. The low energy diets were expected to constitute significant levels of nutritional stress that impair chick development. We aimed to assess if fluctuating asymmetry across the three measured traits consistently reflected the nutritional stress exposure in developing L. fuscus chicks.

\section{Materials and Methods}

\subsection{Experimental Manipulations}

\subsubsection{General Procedure}

Adult Lesser Black-backed Gulls breed between March and August, with each pair usually laying a clutch of 3 eggs [24]. 40 pipping eggs were collected from nests located in the port of Ostend, Belgium, at three different periods in the breeding season: early (31 May 2015), intermediate (7 June 2015) and 
late (14 June 2015). Only one egg was taken per nest, being either the first or second laid egg in order to avoid clutch effects [24]. The eggs were taken to aviary facilities located within the Wildlife Rescue Centre Ostend (VOC Ostend, Belgium), where all the experimental procedures of this study were carried out. The eggs were incubated until hatching (incubator settings: temperature $=37.5^{\circ} \mathrm{C}$, humidity $=62 \%$ ), upon which chicks were randomly assigned to a treatment group.

Newly hatched chicks of the same treatment were housed together under a heat lamp up to the age of 3 days, at which point they were transferred to individual cages where they remained for the rest of the experiment. The cages were $2 \mathrm{~m}$ long by $1 \mathrm{~m}$ wide by $0.5 \mathrm{~m}$ high and partially covered on top and in front by an opaque plastic sheet to provide protection against the rain and wind. These individual cages were all enclosed within a larger flying cage. The chicks were given their respective diets four times a day and were regularly measured and sampled (described ahead). Each chick was subjected to the experimental treatments until it reached the age of 30 days, when chicks of this species usually start to fledge [25]. The experimental manipulations started at the beginning of June (when the first chicks hatched) and continued up to the end of July (when the last chick turned 30 days old).

\subsubsection{Diet Treatments}

In order to induce nutritional stress, the chicks were subjected to treatments of different energy levels via dietary manipulations. The diets consisted of a mix of marine and terrestrial-based food fractions in different proportions, with one treatment receiving $80 \%$ of the marine and $20 \%$ of the terrestrial fraction and the other receiving the reverse. The marine fraction was an equal mix (in dry weight) of Cod (Gadus morhua), Atlantic Mackerel (Scomber scombrus) and Whiting (Merlangius merlangus), whereas the terrestrial fraction was an equal mix (in dry weight) of dried mealworms (Tenebrio molitor larvae), fried potato chips (i.e., crisps) and broiler chicken (Gallus gallus domesticus). The terrestrial fraction was expected to have a higher energy density compared to the marine. Selection of the food items included in each diet was based on previous knowledge of chick diet at the study site, derived from composition analyses of regurgitates [26]. All food was homogenised and embedded in gelatine to avoid selective feeding. Energy intake of the chicks was additionally manipulated by providing a third treatment group with a restricted quantity of the high-energy, $80 \%$ terrestrial food, amounting to the minimum caloric intake per feeding necessary for the growth of a developing chick [27]. Sample size and resource limitations did not allow for a corresponding restriction treatment on the lower energy, marine-based diet.

Three levels of nutritional stress were therefore defined by our treatments (Figure 1), hereafter referred to as (i) Stress1 or "S1" receiving 80\% terrestrial, $20 \%$ marine fraction in an unrestricted amount; (ii) Stress2 or "S2" receiving 20\% terrestrial, $80 \%$ marine fraction in an unrestricted amount; and (iii) Stress 3 or "S3" receiving $80 \%$ terrestrial, $20 \%$ marine fraction in a restricted amount. The S1 treatment was assumed to constitute the least nutritional stress and S3 the most, a ranking which was validated by analysis of body size (at age of 30 days) and the energy ingestion over the duration of the experiment (Figure 1).

Although the chicks were divided among these treatments upon hatching, the full diet regime was imposed on them only from the third day of age onwards. Before that, they were fed a simplified version of their respective diets (cod fillets for S2 and ground chicken for S1 and S3), all in an unrestricted quantity. This was done in deference to ethical guidelines and code of conduct; the first few days after hatching are critical for chicks and introducing the full treatments at this stage could strongly impair their survival. 


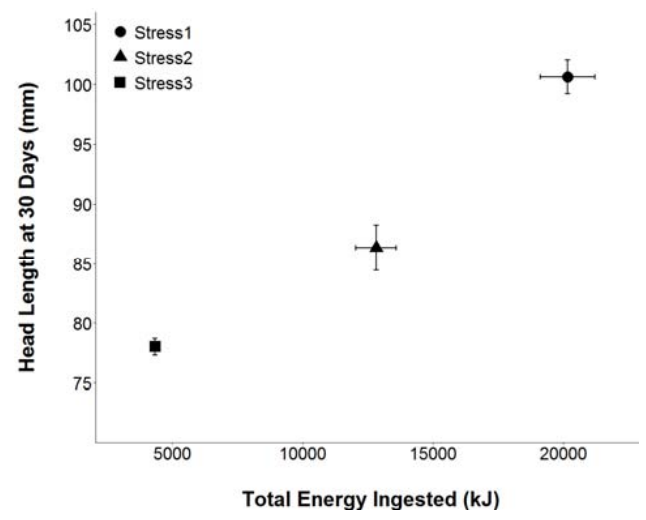

Figure 1. Mean head length at 30 days of age ( $y$-axis) and mean total ingested energy ( $x$-axis) between age 3 and 30 days per treatment ( circle $=\mathrm{S} 1$, triangle $=$ S2 and square $=$ S3). Standard error is given for both axes.

\subsubsection{Measurements}

Length of the head (from the external occipital protuberance to the tip of the bill) was regularly measured for each chick, starting from the day after hatching and following that twice a week (every 3-5 days) up to 30 days of age. Measurements were carried out in duplicate to the nearest $0.01 \mathrm{~mm}$ using a digital calliper. These growth measurements were later regressed on age and the slope was taken as the growth rate for each chick.

Measurements of bilateral traits, for the estimation of fluctuating asymmetry, were conducted at the age of 30 days. The left and right tarsi were measured for each chick from the intertarsal joint up to the base of the middle toe with a digital calliper, in duplicate per side to the nearest $0.01 \mathrm{~mm}$. The left and right wings were measured with a ruler from the distal portion of the carpus up to the tip of the primary feathers, in duplicate to the nearest $1 \mathrm{~mm}$. After these measurements, the 10th primary feather of each wing was plucked and later measured to the nearest $1 \mathrm{~mm}$ by mounting on millimetre paper and straightening the rachis with needles. Feather measurements were done in triplicate, by remounting the apparatus each time to ensure independent measurements.

A final total of 32 chicks were measured for FA. However, due to difficulties in obtaining primary feather samples from smaller sized chicks, particularly of the S3 treatment, the sample size for feather FA was reduced to 23 .

\subsubsection{Animal Welfare}

All the experiments were carried out in accordance with the European/FELASA ethical guidelines for laboratory experimentation in vertebrate animals [28]. In addition, every effort was made to improve the well-being and condition of the hatchlings and minimise their stress. The chicks were housed in indoor enclosures at night under heat lamps until the age of 10 days, or longer if deemed necessary. Individuals that had been exposed to the rain were kept under heat lamps or in incubators until dry. Chicks that showed any sign of illness were removed from the experiment and given veterinary care. At the conclusion of the experiment, the chicks were caged together with the wild gull chicks and juveniles that were under rehabilitation at the VOC care facilities, in order to remove the effect of human imprinting caused by the experiment. When deemed fit, they were released into the wild along with the other juveniles.

\subsection{Data Analyses}

\subsubsection{Fluctuating Asymmetry Estimation}

The level and significance of FA in the measured traits were calculated by the restricted maximum likelihood (REML) estimation of a mixed regression model, as described by Van Dongen et al. [29]. This 
procedure allows the separation of measurement error from bilateral asymmetry analysis. The presence of directional asymmetry-a type of asymmetry that is biased towards one side being larger than the other-was assessed by a $t$-test of the fixed effects, with degrees of freedom corrected for statistical dependence by Satterthwaite formulas. The random intercepts and slopes (both estimated within individuals) estimated the variation in individual trait value and the individual signed FA, respectively, while the random error variance component gave the measurement error. The significance of FA was then calculated by performing a likelihood ratio (LR) test comparing two models: the original, full model and a reduced one without the side as a random effect. Absence of anti-symmetry-a type of asymmetry that occurs in a random direction, producing a bimodal distribution over the population-was verified by testing the signed FA for a normal distribution. The R package lmerTest [30] was used for this mixed model estimation.

In order to increase the statistical power of detecting FA differences, FA values can also be combined across multiple traits [31]. For this method, however, the traits need to be developmentally independent. This was verified by checking the between-trait correlations of signed FA, which were non-significant for all trait pairs $(p>0.05$ for all). FA across the three traits were hence pooled together by treating multiple traits as repeated measurements at the individual level (see [4]). Multi-trait FA estimates were therefore obtained, where every individual had up to three measures of fluctuating asymmetry.

Finally, the absolute, unsigned FA values were obtained and subsequently divided by mean trait size per individual, in order to calculate the asymmetry relative to trait size. The between-trait correlations of these FA values were then tested to check for presence of organism-wide asymmetry.

\subsubsection{Hypothesis Testing}

Relationships with fluctuating asymmetry of individual traits were assessed by linear models ( $\mathrm{lm}$ function in R). FA of each trait was individually modelled as the dependent variable against nutritional stress level (S1, S2 or S3) as the main explanatory variable. Hatching period (early, intermediate or late corresponding to the three collection batches) and growth rate of head length were added in the models as covariates to account for variation in chick quality and in developmental speed caused by the treatment, respectively. Interaction of stress level with hatching period was also included based on a priori expectations [32].

Selection and estimation of explanatory variables were done using Akaike's Information Criterion, with the correction for finite sample size (AICc, [33]). First, a complete set of models with all possible combinations of the above-mentioned parameters was obtained with the dredge function of the $R$ package MuMIn [34]. These models were ranked according to their AICc scores, with the lowest score being the most parsimonious model. Model averaging was applied across the top models (with $\Delta \mathrm{AICc} \leq 2, \triangle \mathrm{AIC}$ being the difference between the AICc score of a model and the best model, [33]). This procedure derives the parameter and error estimates from the weighted averages of these values across the multiple selected models. By performing model averaging, the estimated coefficients of the best explanatory variables and their relative importance (sum of the Akaike weights across all the models in which a particular variable appears) are therefore obtained.

To study relationships with multi-trait FA, we performed mixed regression analysis with repeated measure structure (i.e., treating trait as repeated measures at individual level), with the lme4 package in R [35]. Multi-trait FA estimates were modelled on the same set of variables: stress level, hatching period, their interaction, and growth rate as fixed factors. Individual was added as a random factor to account for the fact that FA values of multiple traits per individual are not statistically independent. Variable selection was performed as before, by averaging of the best models selected using AICc.

All analyses were performed using the public domain software R (Version 3.2.3, [36]) with its integrated development environment software RStudio (Version 0.99.879, [37]). 


\section{Results}

\subsection{FA Estimation}

Estimation of FA by the mixed model produced consistently larger FA variance components as compared to the error component (Table 1), indicating the presence of significant fluctuating asymmetry. This was confirmed by the likelihood ratio test $(p<0.001$ for all traits). The model also revealed the presence of directional asymmetry in the tarsi (Table 1), with the right tarsus tending to be larger than the left in the population. Directional asymmetry was absent for the primary feather and wing. The signed FA values of each trait were normally distributed, confirming the absence of anti-symmetry. Pairwise comparisons (Spearman's rank correlation) of absolute, unsigned FA values produced no significant correlations between traits $(p>0.05$ for all).

Table 1. Descriptive statistics of fluctuating asymmetry estimation. The sample size $(n)$, directional asymmetry (mean (with standard error SE), $t$-statistic (with degrees of freedom df) and $p$-value), random variance components of fluctuating asymmetry $\left(\mathrm{V}_{\mathrm{FA}}\right)$ and measurement error $\left(\mathrm{V}_{\mathrm{ME}}\right)$, and the results of the likelihood ratio test ( $\chi^{2}$ and $p$-value) for each trait are given.

\begin{tabular}{|c|c|c|c|c|c|c|c|c|}
\hline \multirow{2}{*}{ Trait } & \multirow{2}{*}{$n$} & \multicolumn{3}{|c|}{ Directional Asymmetry (DA) } & \multicolumn{2}{|c|}{$\begin{array}{c}\text { Variance } \\
\text { Components }\end{array}$} & \multicolumn{2}{|c|}{$\begin{array}{c}\text { Likelihood Ratio } \\
\text { Test }\end{array}$} \\
\hline & & Mean \pm SE & $t$-Statistic (df) & $p$ & $\mathrm{~V}_{\mathrm{FA}}$ & $\mathrm{V}_{\mathrm{ME}}$ & $\chi^{2}$ & $p$ \\
\hline Feather & 23 & $0.1594 \pm 0.3942$ & $0.404(22.57)$ & 0.690 & 3.48 & 0.14 & 166.90 & $<0.001$ \\
\hline Tarsus & 32 & $0.3702 \pm 0.1780$ & $2.080(31.26)$ & 0.046 & 1.01 & 0.01 & 222.46 & $<0.001$ \\
\hline Wing & 32 & $0.2500 \pm 0.7354$ & $0.340(30.99)$ & 0.736 & 16.74 & 0.56 & 118.47 & $<0.001$ \\
\hline
\end{tabular}

\subsection{Feather FA}

The mean feather FA (corrected for feather size) was highest for the S3 treatment $(0.039 \pm 0.019 \mathrm{~mm}$, Figure 2). AICc analysis produced a single best model within the criterion $(\triangle \mathrm{AICc} \leq 2)$, hence no model averaging was performed. This top model contained stress level, hatching period and their interaction as the parameters explaining the variation in feather FA. The interaction was further examined with pairwise estimates of FA between stress treatments taken per hatching period (Table 2). Stress treatment effects on feather FA seemed to be reduced in intermediate hatched chicks, while early hatched chicks showed increased FA under the highest stress treatment (S3). Late hatched chicks had increased FA under moderate stress (S2) itself; however, feather samples of the late hatched S3 chicks were absent.

Table 2. Modelled estimates, standard error (SE) and upper and lower 95\% confidence intervals (CI) of feather FA for pairwise contrasts of stress (i.e., difference between stress treatments), given per hatching period. Missing values for the late hatching period are due to the absence of feather samples of the late hatched S3 chicks.

\begin{tabular}{ccccc}
\hline Treatment Contrast & Hatching Period & Estimate (SE) & Lower CI & Upper CI \\
\hline S1-S2 & Early & $-0.0121(0.0067)$ & -0.0296 & 0.0055 \\
S1-S3 & Early & $-0.0635(0.0101)$ & -0.0898 & -0.0373 \\
S2-S3 & Early & $-0.0515(0.0107)$ & -0.0792 & -0.0238 \\
S1-S2 & Intermediate & $-0.0069(0.0067)$ & -0.0244 & 0.0106 \\
S1-S3 & Intermediate & $-0.0133(0.0084)$ & -0.0351 & 0.0086 \\
S2-S3 & Intermediate & $-0.0063(0.0077)$ & -0.0264 & 0.0137 \\
S1-S2 & Late & $-0.0563(0.0107)$ & -0.0840 & -0.0286 \\
S1-S3 & Late & $\backslash$ & $\backslash$ & $\backslash$ \\
S2-S3 & Late & $\backslash$ & $\backslash$ & $\backslash$ \\
\hline
\end{tabular}




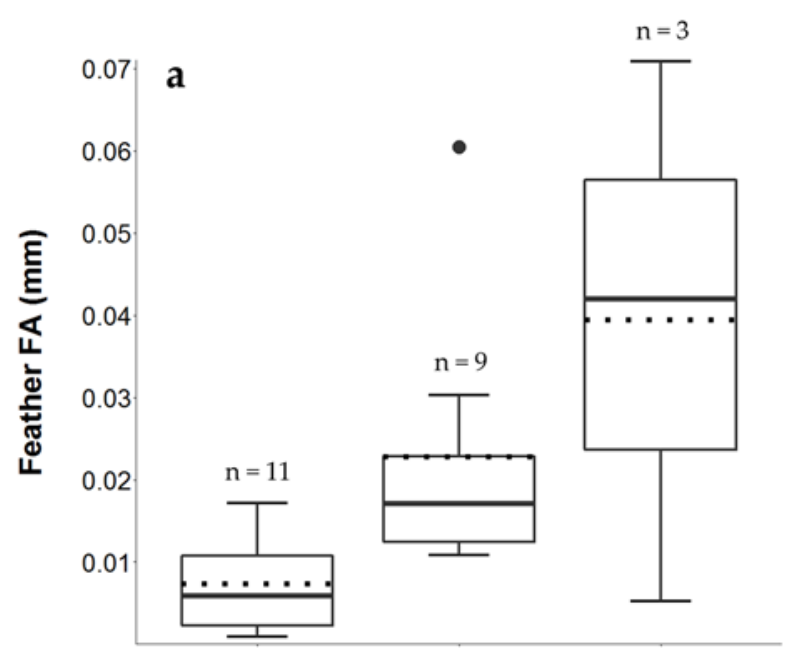

(a)

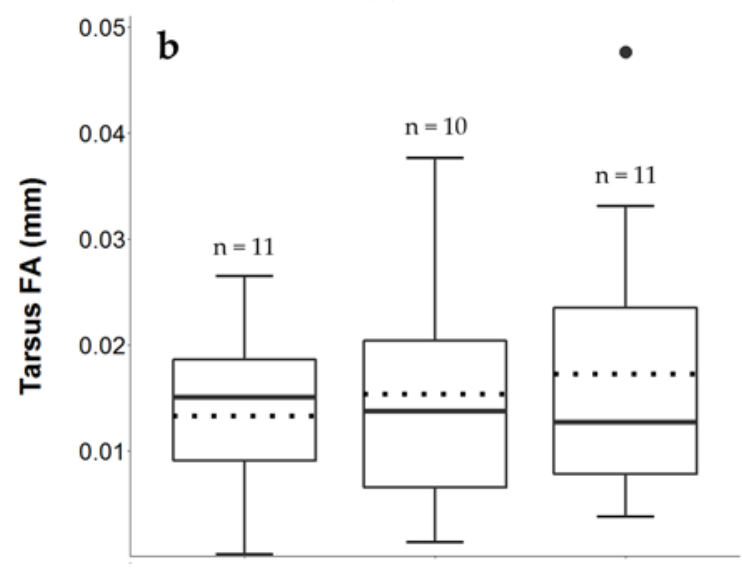

(b)

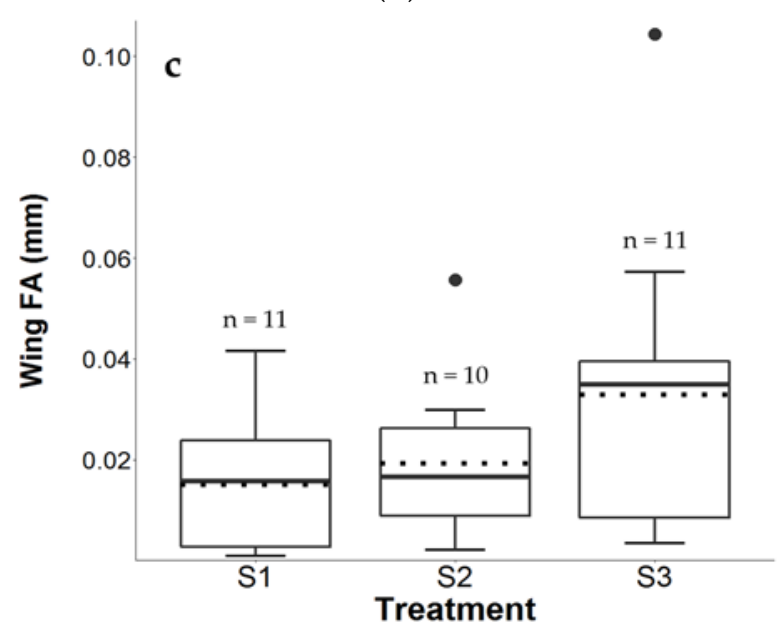

(c)

Figure 2. Size-corrected FA values of the feather (a); tarsus (b); and wing (c) per stress treatment. Mean (dotted line), median (solid line) and 1st and 3rd quartile (lower and upper hinges of the box) of each treatment are shown. The whiskers correspond to the lowest and highest values that are within 1.5 times the inter-quartile range; values outside this range are represented by points. Sample size $(n)$ of each treatment is given above the box. 


\subsection{Tarsus $F A$}

FA of the tarsus did not show large variations among treatments (Figure 2). Averaging of the top three models for tarsus FA retained all model parameters, each having a low importance however (Relative variable importance: Stress $=0.24$, Hatching Period $=0.24$, Growth Rate $=0.28$, Stress*Hatching Period $=0.24$ ). As before, the interaction was examined with pairwise estimates per hatching period (Table 3). FA did not show a clear and consistent relationship with stress across the hatching periods. Early hatched chicks appeared to have increased FA levels only with high stress (S3), whereas late hatched chicks showed increased FA at moderate stress levels (S2), with no significant difference between lowest and highest stress treatments (S1 and S3). Intermediate hatched chicks did not differ in FA in relation to stress levels (Table 3).

Table 3. Modelled estimates, standard error (SE) and upper and lower 95\% confidence intervals (CI) of tarsus FA for pairwise contrasts of stress (i.e., difference between stress treatments), given per hatching period.

\begin{tabular}{ccccc}
\hline Treatment Contrast & Hatching Period & Estimate (SE) & Lower CI & Upper CI \\
\hline S1-S2 & Early & $0.0072(0.0133)$ & -0.0262 & 0.0406 \\
S1-S3 & Early & $-0.0325(0.0193)$ & -0.0809 & 0.0160 \\
S2-S3 & Early & $-0.0397(0.0140)$ & -0.0749 & -0.0044 \\
S1-S2 & Intermediate & $-0.0002(0.0127)$ & -0.0320 & 0.0316 \\
S1-S3 & Intermediate & $-0.0034(0.0199)$ & -0.0533 & 0.0465 \\
S2-S3 & Intermediate & $-0.0032(0.0111)$ & -0.0311 & 0.0246 \\
S1-S2 & Late & $-0.0229(0.0229)$ & -0.0805 & 0.0347 \\
S1-S3 & Late & $0.00001(0.0227)$ & -0.0571 & 0.0571 \\
S2-S3 & Late & $0.0229(0.0106)$ & -0.0037 & 0.0494 \\
\hline
\end{tabular}

\subsection{Wing $F A$}

The highest mean wing FA (size-corrected) was again found in the S3 treatment $(0.033 \pm 0.009 \mathrm{~mm}$, Figure 2). Wing FA values were square root transformed prior to analysis in order to meet the assumptions of normality and homoscedasticity of model residuals. The average of the top three models contained stress, hatching day and growth rate as the explanatory variables (Relative variable importance: Stress $=0.25$, Hatching Period $=0.76$, Growth Rate $=0.75$ ). FA appeared to have a positive relationship with stress (Table 4); confidence intervals, however, indicate non-significant effects.

Table 4. Modelled estimates, standard error (SE) and upper and lower 95\% confidence intervals (CI) of wing FA for pairwise contrasts of stress (i.e., difference between stress treatments). Values are averaged over the hatching period here, as the stress-hatching period interaction was not retained as a model parameter.

\begin{tabular}{cccc}
\hline Treatment Contrast & Estimate (SE) & Lower CI & Upper CI \\
\hline S1-S2 & $-0.0238(0.0567)$ & -0.1648 & 0.1172 \\
S1-S3 & $-0.0693(0.0803)$ & -0.2689 & 0.1303 \\
S2-S3 & $-0.0456(0.0385)$ & -0.1414 & 0.0502 \\
\hline
\end{tabular}

\subsection{Multi-Trait FA}

FA values were combined across traits to study relationships with multi-trait FA estimates. AICc analysis yielded a single best model, which was the intercept model. Hence, none of the explanatory variables were selected to explain variation in FA when combined across traits. 


\section{Discussion}

The central hypothesis tested in our study was that increased levels of nutritional stress (induced by energetic constraints) would result in more asymmetric growth at the organism-wide level. Chicks of the most stressed treatment (S3) did indeed have the highest levels of FA overall (Figure 2), and statistical analysis showed that FA was associated positively with stress for the feather, in accordance with our predictions. However, we were unable to establish conclusive, linear relationships between stress and FA for the wings, tarsi and multi-trait estimates. Lack of concordance between traits was further seen in the absence of between-trait correlations in unsigned FA values. Therefore, while the degree of fluctuating asymmetry does appear to reflect energetic stress exposure in developing chicks, FA-stress relationships were not uniform across traits.

The tarsus is a relatively well-studied trait for fluctuating asymmetry in birds, and has been previously linked to a number of different stressors (see $[38,39]$ for examples). The similar levels of tarsus FA between our stress treatments (Figure 2), and its mixed and mostly non-significant relationships with stress across the different hatching periods (Table 3) are therefore surprising and contrary to expectations. Furthermore, although wing FA showed a positive relationship with stress, confidence intervals indicate that this relationship is unreliable (Table 4). This heterogeneity between traits likely resulted in the failure to detect any FA-stress relationships using the multi-trait approach. Trait-specific asymmetry patterns are in agreement with previous findings (see $[11,12])$ and may be attributed to several (non-mutually exclusive) causes.

First and foremost, different traits can have variable responses to stress. For instance, the compensational growth hypothesis (sensu [40]) proposes that the stage of ontogeny at which a trait is exposed to stress can influence the manifestation of FA across it. According to this hypothesis, regulatory mechanisms between the two sides of a trait work to restore trait symmetry towards the end of development. Among the studied traits, the tarsi were fully grown (or close to) in most chicks at the time of measurement, which means that compensational growth could have restored their bilateral symmetry. Conversely, the wings and primary feathers were still developing at the end of our experiment and hence would have been more likely to be asymmetrical. However, compensational growth is just one of the mechanisms proposed to describe the ontogeny of FA, and many studies have found evidence contrary to it (see [41,42] for examples).

Similarly, heterogeneous trait responses may be explained by the concept of "windows of opportunity" [43]. A trait is said to be vulnerable to environmental stressors only during certain developmental stages, leading to the production of visible aberrant phenotypes. These windows of opportunity may vary among traits [43,44]; the tarsi and wings may have experienced only restricted levels of energetic stress during the vulnerable period of their development. However, given that stress exposure was nearly constant throughout our experiment, this is not a very likely explanation of our results. Alternatively, Aparicio and Bonal [45] proposed that the structural composition of a trait may affect relationships between stress and FA as well. In particular, traits with more structural components per unit length may be less likely to manifest fluctuating asymmetry.

Trait-specific susceptibility to FA may also be related to the functional importance of a trait $[10,44,46]$. Traits of higher functional value should be subjected to stronger stabilizing selection, as minor reductions in their symmetry can impose a substantial fitness cost. These will therefore be less susceptible to developmental perturbations [10]. For instance, Gummer and Brigham [47] found lower degrees of asymmetry in traits having a higher role in mobility in Little Brown Bats (Myotis lucifugus). All traits measured in our study were related to mobility, i.e., tarsi for walking and wing and feathers for flight. However, the primary feathers and wings may have been of relatively lower functional importance in young chicks yet to fledge, as compared to tarsi. If so, the latter can be expected to be more strongly buffered against the nutritional stress, hence weakening the FA-stress relationships. Furthermore, Boonekamp et al. [48] have suggested that functional traits that are sensitive to developmental conditions may still be poor fitness predictors, given that their development is likely to be more strongly canalized. 
Finally, inconsistent results may arise from problems with statistical analysis or experimental design. We acknowledge that our sample sizes (Figure 2) were on the low side for study of relationships with fluctuating asymmetry, and the latter should therefore be interpreted with some caution. In particular, the feather sample size of the S3 treatment was very small (three individuals) as compared to the other groups, which may have biased the analysis of this trait. However, we believe that, overall, our statistical analyses were biologically and statistically sound. FA variance components were significantly larger than the error components in the mixed model estimation (Table 1), indicating the absence of measurement bias. This model also accounted for directional asymmetry, which may otherwise confound the FA estimation [29]. Our results are also unlikely to be compromised by experimental flaws, total energy ingestions and body sizes of the chicks (Figure 1) verify that our treatments were sufficient to induce different stress levels. Moreover, being an experimental study, it is less likely to be confounded by unknown environmental factors. Therefore, the heterogeneous FA-stress relationships in our results appear to be at least partly real, rather than artefacts.

\section{Conclusions}

Our study showed increasing levels of FA with nutritional stress exposure in developing Lesser Black-backed Gull hatchlings, demonstrating its potential as marker of stress. The increased feather asymmetry can have direct consequences on flight performance [49], and may be indicative of a potentially impaired future fitness. However, the heterogeneity seen across different traits and apparent absence of organism-wide asymmetry indicate that FA does not consistently reflect developmental stability or fitness. Its success as a stress indicator may therefore depend on the 'correct' trait(s) being studied. Further experimental research remains necessary to understand and explain the manifestation of stress-induced developmental instability as FA in different traits before it can be considered a reliable biomarker.

Acknowledgments: We are indebted to the Port Authority of Ostend for providing access to the breeding sites of the gulls, and the Wildlife Rescue Centre Ostend (VOC), the Flanders Marine Institute (VLIZ) and Ghent University for providing the infrastructure to conduct the experiment. We also thank Sam Durnez and Sergio Delgado, Masters' students who assisted in the preparation and undertaking of the experiment, as well as the staff and volunteers at the VOC. This research was supported by Research Foundation-Flanders (FWO) grant G0E1614N to W.M., L.L. and L.D.N. C.S.A.S. is funded by a grant from the Special Research Fund of Ghent University (B/13833/01-BOF13/DOC/034), while A.S. is funded by an Fundação para a Ciência e a Tecnologia grant (FCT, Ministry of Science, Technology and Higher Education, Portugal, Reference PB/BD/113792/2015) in the framework of the Biology and Ecology of Global Change (BEGC) doctoral program.

Author Contributions: T.G., C.S.A.S. and A.S. conceived, designed and performed the experiments; T.G., C.S.A.S. and L.L. analyzed the data; T.G. and C.S.A.S. wrote a first draft of the paper, and all authors contributed to discussing and writing subsequent drafts.

Conflicts of Interest: The authors declare no conflict of interest. The founding sponsors had no role in the design of the study; in the collection, analyses, or interpretation of data; in the writing of the manuscript, and in the decision to publish the results.

\section{References}

1. Adams, S.M.; Giesy, J.P.; Tremblay, L.A.; Eason, C.T. The use of biomarkers in ecological risk assessment: Recommendations from the Christchurch conference on Biomarkers in Ecotoxicology. Biomarkers 2001, 6, 1-6. [CrossRef] [PubMed]

2. Tomkins, J.L.; Andrews, S. Fluctuating Asymmetry. Encycl. Life Sci. 2001. [CrossRef]

3. Palmer, A.R. Fluctuating asymmetry analyses: A primer. In Developmental Instability: Its Origins and Evolutionary Implications; Springer: Dordrecht, The Netherlands, 1994; Volume 93, pp. 335-364.

4. Lens, L.; Dongen, S.; Kark, S.; Matthysen, E. Fluctuating asymmetry as an indicator of fitness: Can we bridge the gap between studies? Biol. Rev. 2002, 77, 27-38. [CrossRef] [PubMed]

5. Leary, R.F.; Allendorf, F.W. Fluctuating asymmetry as an indicator of stress: Implications for conservation biology. Trends Ecol. Evol. 1989, 4, 214-217. [CrossRef]

6. Moller, A.P. Developmental stability and fitness: A review. Am. Nat. 1997, 149, 916-932. [CrossRef] [PubMed] 
7. Clarke, G.M. Fluctuating asymmetry of invertebrate populations as a biological indicator of environmental quality. Environ. Pollut. 1993, 82, 207-211. [CrossRef]

8. Palmer, A.R. Waltzing with asymmetry: Is fluctuating asymmetry a powerful new tool for biologists or just an alluring new dance step? Bioscience 1996, 46, 518-532. [CrossRef]

9. Clarke, G.M.; McKenzie, L.J. Fluctuating asymmetry as a quality control indicator for insect mass rearing processes. J. Econ. Entomol. 1992, 85, 2045-2050. [CrossRef]

10. Clarke, G.M. Relationships Between Developmental Stability and Fitness: Application for Conservation Biology. Conserv. Biol. 1995, 9, 18-24. [CrossRef]

11. Lens, L.; Eggermont, H. Fluctuating asymmetry as a putative marker of human-induced stress in avian conservation. Bird Conserv. Int. 2008, 18, S125-S143. [CrossRef]

12. Bjorksten, T.A.; Fowler, K.; Pomiankowski, A. What does sexual trait FA tell us about stress? Trends Ecol. Evol. 2000, 15, 163-166. [CrossRef]

13. Leung, B.; Forbes, M.R. Fluctuating asymmetry in relation to stress and fitness Effects of trait type as revealed by meta-analysis. Ecoscience 1996, 3, 400-413. [CrossRef]

14. Swaddle, J.P.; Witter, M.S. Food, Feathers and Fluctuating Asymmetries. R. Soc. 1994, 255, 147-152. [CrossRef]

15. Nilsson, J. Energetic stress and the degree of fluctuating asymmetry: Implications for a long-lasting, honest signal. Evol. Ecol. 1994, 8, 248-255. [CrossRef]

16. Grieco, F. Greater food availability reduces tarsus asymmetry in nestling blue tits. Condor 2003, 105, 599-603. [CrossRef]

17. Sillanpää, S.; Salminen, J.-P.; Eeva, T. Fluctuating asymmetry in great tit nestlings in relation to diet quality, calcium availability and pollution exposure. Sci. Total Environ. 2010, 408, 3303-3309. [CrossRef] [PubMed]

18. Vangestel, C.; Lens, L. Does fluctuating asymmetry constitute a sensitive biomarker of nutritional stress in house sparrows (Passer domesticus)? Ecol. Indic. 2011, 11, 389-394. [CrossRef]

19. Regulation, E.C. No. 1380/2013 of the European Parliament and of the Council of 11 December 2013 on the Common Fisheries Policy, Amending Council Regulations (EC) No. 1954/2003 and (EC) No. 1224/2009 and repealing Council Regulations (EC) No. 2371/2002 and (EC). Off. J. Eur. Union 2013, 354, $22-61$.

20. Bicknell, A.W.J.; Oro, D.; Camphuysen, K.C.J.; Votier, S.C. Potential consequences of discard reform for seabird communities. J. Appl. Ecol. 2013, 50, 649-658. [CrossRef]

21. Sommerfeld, J.; Mendel, B.; Fock, H.O.; Garthe, S. Combining bird-borne tracking and vessel monitoring system data to assess discard use by a scavenging marine predator, the lesser black-backed gull Larus fuscus. Mar. Biol. 2016, 163, 1-11. [CrossRef]

22. Hume, R. Complete Birds of Britain and Europe, 1st ed.; Dorling Kindersley: London, UK, 2002.

23. Isaksson, N.; Evans, T.J.; Shamoun-Baranes, J.; Åkesson, S. Land or sea? Foraging area choice during breeding by an omnivorous gull. Mov. Ecol. 2016, 4, 1-14. [CrossRef] [PubMed]

24. Harris, M.P. Aspectes of the breeding biology of the gulls Larus argentatus, L. fuscus and L. marinus. Ibis 1964, 106, 432-456. [CrossRef]

25. Del Hoyo, J.; Elliott, A.; Sargatal, J. Handbook of the Birds of the World, Vol. 3: Hoatzin to Auks; Lynx Edicions: Barcelona, Spain, 1996.

26. Research Institute for Nature and Forest (INBO), Brussels, Belgium. Unpublished work. 2012.

27. Sotillo, A.; Ghent University, Ghent, Belgium. Unpublished work. 2014.

28. Smith, J.A.; van den Broek, F.A.R.; Martorell, J.C.; Hackbarth, H.; Ruksenas, O.; Zeller, W. Principles and practice in ethical review of animal experiments across Europe: Summary of the report of a FELASA working group on ethical evaluation of animal experiments. Lab. Anim. 2007, 41, 143-160. [CrossRef] [PubMed]

29. Van Dongen, S.; Molenberghs, G.; Matthysen, E. The statistical analysis of fluctuating asymmetry: REML estimation of a mixed regression model. J. Evol. Biol. 1999, 12, 94-102. [CrossRef]

30. Tests in Linear Mixed Effects Models, version 2.0-32; lmerTest; Danmarks Tekniske Universitet: Copenhagen, Denmark, 2016.

31. Leung, B.; Forbes, M.; Houle, D. Fluctuating Asymmetry as a Bioindicator of Stress: Comparing Efficacy of Analyses Involving Multiple Traits. Am. Nat. 2000, 155, 101-115. [CrossRef] [PubMed]

32. Palestis, B.G. Fluctuating Asymmetry in Common Tern (Sterna Hirundo) Chicks Varies with Hatching Order and Clutch Size. Auk 2009, 126, 815-822. [CrossRef]

33. Burnham, K.P.; Anderson, D.R. Model Selection and Multimodel Inference: A Practical Information-Theoretic Approach, 2nd ed.; Springer: New York, NY, USA, 2002. 
34. Multi-Model Inference (MuMIn), version 1.15.6; R Foundation for Statistical Computing: Vienna, Austria, 2015.

35. Bates, D.; Maechler, M.; Bolker, B.; Walker, S. Fitting Linear Mixed-Effects Models Using Ime4. Statistics 2015, arXiv:1406.5823.

36. R: A Language and Environment for Statistical Computing; R Foundation for Statistical Computing: Copenhagen, Denmark, 2015.

37. RStudio Team. RStudio: Integrated Development for R; RStudio: Boston, MA, USA, 2015.

38. Lens, L.; Van Dongen, S.; Matthysen, E. Fluctuating asymmetry as an early warning system in the critically endangered Taita Thrush. Conserv. Biol. 2002, 16, 479-487. [CrossRef]

39. Maul, J.D.; Farris, J.L. Monitoring Exposure of Northern Cardinals, Cardinalis Cardinalis, to Cholinesterase-Inhibiting Pesticides: Enzyme Activity, Reactivations, and Indicators of Environmental Stress. Environ. Toxicol. 2005, 24, 1721-1730. [CrossRef]

40. Swaddle, J.P.; Witter, M.S. On the ontogeny of developmental stability in a stabilized trait. Proc. R. Soc. Lond. Ser. B Biol. Sci. 1997, 264, 329-334. [CrossRef]

41. Chippindale, A.K.; Palmer, A.R. Persistence of subtle departures from symmetry over multiple molts in individual brachyuran crabs: Relevance to developmental stability. Genetica 1993, 89, 185-199. [CrossRef]

42. Aparicio, J.M. Patterns of fluctuating asymmetry in developing primary feathers: A test of the compensational growth hypothesis. Proc. R. Soc. Lond. Ser. B Biol. Sci. 1998, 265, 2353-2357. [CrossRef]

43. Clarke, G.M. The genetic basis of developmental stability. IV. Individual and population asymmetry parameters. Heredity 1998, 80, 553-561. [CrossRef]

44. Rasmuson, M. Fluctuating asymmetry-Indicator of what? Hereditas 2002, 136, 177-183. [CrossRef] [PubMed]

45. Aparicio, J.M.; Bonal, R. Why do some traits show higher fluctuating asymmetry than others? A test of hypotheses with tail feathers of birds. Heredity 2002, 89, 139-144. [CrossRef] [PubMed]

46. Palmer, A.R.; Strobeck, C. Fluctuating Asymmetry: Measurement, Analysis, Patterns. Annu. Rev. Ecol. Syst. 1986, 17, 391-421. [CrossRef]

47. Gummer, D.L.; Brigham, R.M. Does fluctuating asymmetry reflect the importance of traits in little brown bats (Myotis lucifugus)? Can. J. Zool. 1995, 73, 990-992. [CrossRef]

48. Boonekamp, J.J.; Dijkstra, R.; Dijkstra, C.; Verhulst, S. Canalization of development reduces the utility of traits as fitness biomarkers: Feather fault bars in nestling birds. Funct. Ecol. 2016. [CrossRef]

49. Thomas, A.L. The aerodynamic costs of asymmetry in the wings and tail of birds: Asymmetric birds can't fly round tight corners. Proc. R. Soc. Lond. B Biol. Sci. 1993, 1341, 181-189. [CrossRef] 